Arq. Bras. Med. Vet. Zootec., v.61, n.2, p.523-526, 2009

\title{
Comunicação
}

[Communication]

\section{Luxação de patela em cães: estudo retrospectivo}

\author{
[Patellar luxation in dogs: a retrospective study]
}

\author{
M.M.D. Souza ${ }^{1}$, S.C. Rahal $^{2}$, C.C. Otoni $^{3}$, A.C. Mortari ${ }^{3}$, S.E.R.S. Lorena ${ }^{3}$ \\ ${ }^{1}$ Aluno de graduação - FMVZ-UNESP - Botucatu, SP \\ ${ }^{2}$ Faculdade de Medicina Veterinária e Zootecnia - UNESP \\ Distrito de Rubião Júnior $\mathrm{s} / \mathrm{n}$ \\ 18618-000 - Botucatu, SP \\ ${ }^{3}$ Aluno de pós-graduação - FMVZ-UNESP - Botucatu, SP
}

A luxação de patela é uma das mais comuns anormalidades que acomete o joelho dos cães (Horne, 1971; Hulse, 1981; Tomlinson e Constantinescu, 1994). A afecção pode ser congênita, também referida como de desenvolvimento, ou traumática, sendo a luxação de patela medial congênita a mais frequentemente observada (Hulse, 1981; Hayes et al., 1994; Arnoczky e Tarvin, 1998). A fisiopatologia da luxação congênita não está inteiramente compreendida, visto haver poucos dados objetivos para sugerir quais das deformidades associadas contribuem para a indução da luxação e quais desenvolvem como consequência do deslocamento patelar (L'Eplattenier e Montavon, 2002). A intensidade das deformidades depende da severidade da luxação patelar e da idade do animal (Johnson e Hulse, 2002). Outro fator importante é a permanência da luxação; quanto mais tempo as forças anormais atuarem na placa fisária de um cão jovem, maiores serão as alterações angulares e de torção (Roush, 1993).

Os sinais clínicos variam com o grau de luxação e incluem claudicação intermitente ou consistente, defeitos conformacionais, dor e relutância em se mover (Roush, 1993). O diagnóstico é baseado na palpação do joelho afetado, contudo o exame radiográfico é útil para documentar o grau de deformidade do membro tão bem quanto o grau de osteoartrite presente na articulação do joelho (Johnson e Hulse, 2002; L'Eplattenier e Montavon, 2002). O tratamento é dependente do grau da luxação, sendo em sua maioria realizado por meio de procedimentos cirúrgicos de reconstrução dos tecidos moles e ósseos (Horne, 1971; Read, 1999; Denny e Butterworth, 2000). Entretanto, independente das técnicas, o objetivo é conseguir que a patela se posicione adequadamente no sulco troclear e, assim, permaneça durante toda a amplitude do movimento (Read, 1999).

Visto os achados epidemiológicos contribuírem na caracterização de uma doença e auxiliarem no diagnóstico, prevenção e determinação dos tratamentos mais apropriados, o presente trabalho teve por objetivo realizar estudo retrospectivo de cães portadores de luxação patelar, submetidos ou não a procedimentos cirúrgicos corretivos.

Avaliaram-se retrospectivamente 75 cães portadores de luxação patelar, atendidos no Serviço de Cirurgia da FMVZ-UNESP Botucatu, durante cinco anos (2001-2006). Foram pesquisados dados referentes à raça, sexo, idade e peso dos cães no momento do atendimento, histórico com observações referentes ao tipo de sinais clínicos e momento da constatação da afecção pelo proprietário. O grau de luxação patelar foi determinado pelo exame clínico, segundo classificação de Roush

Recebido em 17 de junho de 2008

Aceito em 26 de março de 2009

* Autor para correspondência (corresponding author)

E-mail: sheilacr@fmvz.unesp.br 
(1993). Os membros acometidos, o tipo de procedimento cirúrgico efetuado, e a presença ou não de outra afecção ortopédica detectada pelo exame físico ou radiográfico foram também investigados. Os dados obtidos foram avaliados por estudo de associação e teste exato de Fisher. $\mathrm{O}$ limite de significância estatística foi $\mathrm{P}<0,05$.

De um total de 75 cães (n=122 membros acometidos), 62 (82,7\%) tinham luxação medial e $11(14,7 \%)$ luxação lateral. Os achados assemelham-se aos citados por outros autores (DeAngelis e Hohn, 1970; L'Eplattenier e Montavon, 2002), que afirmaram ser de $75 \%$ ou mais os casos relacionados à luxação patelar medial e menos de $25 \%$, à lateral. Apenas ocasionalmente as luxações lateral e medial ocorrem simultaneamente na mesma articulação (Horne, 1971; L'Eplattenier e Montavon, 2002). No presente estudo, isso foi observado em dois casos $(2,7 \%)$ - um cão da raça Chow-Chow (30kg de peso) e outro sem raça definida (6kg de peso) - e afetava apenas um membro. Em nenhum cão foram detectados membros com luxações opostas.

A luxação medial é uma afecção que ocorre especialmente em cães de raças toy e miniaturas como Poodle, Yorkshire Terrier, Chihuahua, Pomerânia, Pequinês, Boston Terrier (DeAngelis e Hohn, 1970; Priester, 1972; Hulse, 1981; Hayes et al., 1994; LaFond et al., 2002). Nos casos de luxação medial, $5(6,7 \%)$ não tinham raça definida (peso médio de 4,8kg) e $57(76 \%)$ pertenciam a padrões raciais definidos, sendo a maior prevalência em Poodles, 27 (36\%), Pinschers, 13 (17,3\%) e Yorkshires, 4 (5,3\%). As demais raças apareceram com um ou dois representantes cada.

Na luxação lateral, um cão $(1,3 \%)$ não tinha raça definida (peso médio de $14,5 \mathrm{~kg})$ e $10(13,3 \%)$ pertenciam a padrões raciais definidos, com maior prevalência de Poodles, 16 (8\%), Pinschers, $2(2,7 \%)$. As demais raças apareceram com um ou dois representantes cada. Estes valores diferiram dos citados por outros autores, que referiram ser a luxação patelar lateral de maior frequência em cães de raças grandes, como o São Bernardo, Malamute e Setter Irlandês (Hulse, 1981; Alam et al., 2007). Contudo, devese considerar que a maioria dos cães trazidos para atendimento no Hospital Veterinário da FMVZ incluía raças de porte médio a pequeno.
Segundo Roush (1993), na luxação de patela medial, as fêmeas são mais afetadas que os machos, ambos os membros podem ser afetados e $50 \%$ tem envolvimento bilateral. No presente estudo, ocorreram mais casos em fêmeas que em machos $(\mathrm{P}=0,005)$, tanto na luxação medial como na lateral. Segundo Priester (1972) e Alam et al. (2007), o risco de fêmeas apresentarem a luxação patelar é uma vez e meia ou mais que em machos. Dos 62 cães com luxação medial, 20 $(32,3 \%)$ tinham a lesão unilateral e $42(67,7 \%)$ bilateral. Dos 11 cães com luxação lateral, cinco (45,5\%) apresentaram afecção unilateral e seis $(54,5 \%)$, bilateral. Hayes et al. (1994), em estudo retrospectivo de 10 anos, incluindo duas instituições americanas, constatou que, de 124 casos de luxação patelar canina, as bilaterais $(\mathrm{n}=80)$ foram mais comuns que as unilaterais $(n=44)$.

A etiologia específica da luxação patelar não está totalmente esclarecida (Hulse, 1981; Read, 1999; Denny e Butterworth, 2000). Na maioria dos casos, a lesão é considerada congênita ou de desenvolvimento, mas pode também ser de origem traumática (Horne, 1971; Denny e Butterworth, 2000). O histórico e a presença de outros sinais clínicos, tais como lacerações, abrasões, fraturas, podem auxiliar no diagnóstico da luxação patelar traumática (Roush, 1993). Em $24(32 \%)$ dos casos, os cães foram trazidos para atendimento com idade variando de dois meses a um ano (média de 8,26 meses). Destes, 21 (28\%) apresentaram sinais clínicos de claudicação e dificuldades de locomoção e apoio do membro desde jovens, sugerindo processo congênito, Três (4\%) foram trazidos devido a trauma. Cinquenta e um cães $(68 \%)$ tinham entre 14 meses e nove anos de idade, sendo que em $12 \%$ havia histórico de possível trauma e em 56\% o histórico clínico era inconsistente. Nestes últimos foram considerados sugestivos de processo congênito a presença de luxação bilateral e deformidades ósseas características da afecção. No estudo de Hayes et al. (1994), 82\% dos casos tinham origem congênita; $15 \%$, adquirida e em 3\% a origem não pode ser determinada.

A ruptura do ligamento cruzado tem sido observada em cães com luxação de patela medial (De Angelis e Hohn, 1970; Willauer e Vasseur, 1987). Contudo, segundo Hayes et al. (1994), a prevalência de ruptura do ligamento cruzado cranial não difere entre cães com luxação patelar 
com outras condições ortopédicas. De todos os casos avaliados no presente estudo, em apenas dois o exame ortopédico indicou movimento de gaveta cranial, porém a suspeita de ruptura do ligamento cruzado cranial não foi confirmada cirurgicamente.

O deslocamento da patela é apenas uma das anormalidades presentes na luxação patelar, visto haver numerosas alterações músculo-esqueléticas primárias ou secundárias (Tomlinson e Constantinescu, 1994). De acordo com o tipo e severidade das anormalidades, a luxação patelar pode ser classificada em graus I, II, III e IV (Tomlinson e Constantinescu, 1994; Read, 1999; Denny e Butterworth, 2000). No presente estudo, nos 62 casos de luxação medial, a maior frequência foi a de grau II (25 casos, 33,3\%), seguida pelos graus III (20 casos; 26,7\%), IV (11 casos; $14,7 \%)$ e I $(6 ; 8 \%)$. Nas luxações laterais, a maior frequência foi a de grau II (5 casos; 6,7\%), seguido pelo I (4 casos; 5,3\%) e pelos graus III $(1,3 \%)$ e IV $(1,3 \%)$. Considerando ambos os tipos de luxações, o grau de maior expressão entre as fêmeas foi o II (14 casos;18,4\%). Entre os machos, foram, igualmente, II e III $(23,7 \%)$. No estudo de Hayes et al. (1994), as luxações de graus II e III foram também as mais comumente observadas em todas as categorias, raças ou portes. Segundo esses autores, é possível que cães com graus I e eventualmente II nunca tenham sido trazidos para avaliação ou mesmo a luxação não tenha sido detectada visto os discretos sinais clínicos.

Dos 75 cães, $64(85,3 \%)$ foram tratados cirurgicamente e os demais conservativamente com condroprotetores ou a combinação desses com anti-inflamatórios,por serem em sua maioria portadores de outras afecções sistêmicas, tais como neoplasia mamária, cinomose e problemas dermatológicos, entre outras. Salienta-se que, em estudo comparando dois condroprotetores em modelo de osteoartrite canina, observou-se que o sulfado de condroitina estimulou a atividade condrocítica e o hialuronato de sódio não foi capaz de diminuir o processo degenerativo (Gonçalves et. al., 2008). Conforme citado por Denny e Butterworth (2000), o tratamento conservador deve ser limitado aos casos em que a instabilidade não está associada a qualquer sinal clínico ou se a claudicação é infrequente.

Há vários métodos cirúrgicos para o tratamento da luxação patelar e a escolha depende da gravidade da lesão, ou mesmo da preferência do cirurgião (Horne, 1971; Willauer e Vasseur, 1987; Hayes et al., 1994; Read, 1999). Geralmente são realizadas combinações de técnicas para se obter melhores resultados (Horne, 1971; Hulse, 1981; Hayes et al., 1994; Read, 1999; Denny e Butterworth, 2000; Alam et al., 2007). Essa conduta foi adotada nos cães mencionados no presente relato, já que, nos casos de graus I e II, o tratamento utilizado foi a técnica de superposição do retináculo lateral combinada ou não à trocleoplastia. Adicionalmente, a transposição da crista da tíbia foi efetuada em alguns joelhos com grau II. Nos casos de graus III e IV, os métodos incluíram a superposição do retináculo lateral, a trocleoplastia, a desmotomia, a liberação do quadríceps, a transposição da crista da tíbia e, eventualmente, a sutura fabela-patela. Além disso, devido à severidade da lesão, em um cão com grau IV, foi incluída a osteotomia do fêmur. A evolução clínica dos métodos utilizados não foi pesquisada por não ser objeto do presente estudo.

Segundo Denny e Butterworth (2000), há vários indícios de que a luxação congênita tenha um componente hereditário, sendo provavelmente poligênica e multifatorial. Dessa forma, os animais afetados não devem ser colocados em reprodução para evitar a propagação da lesão. $\mathrm{O}$ fato foi alertado aos proprietários, visto que os cães na sua quase totalidade não haviam sido submetidos à esterilização quando do primeiro atendimento.

Com base na análise dos dados fornecidos por este estudo, pode-se dizer que, entre os cães com luxação patelar, o perfil mais comum é de fêmea, raça de pequeno porte, portadora de luxação medial congênita.

Palavras-chave: cão, joelho, luxação de patela 


\begin{abstract}
A five-year retrospective study of dogs with patellar luxation was performed. A total of 75 dogs $(n=122$ limbs) were studied: $82.7 \%(n=62)$ had medial luxation, $14.6 \%(n=11)$ had lateral luxation, and $2.7 \%$ $(n=2)$ had both luxations in same stifle joint. Considering the dogs with medial patellar luxation, $6.7 \%$ $(n=5)$ were mixed-breed and $76 \%(n=57)$ were purebred, with high prevalence in Poodles $(n=27 ; 35.6 \%)$, Pinschers $(n=13 ; 17.3 \%)$, and Yorkshires $(n=4 ; 5.3 \%)$. Of the dogs with lateral patellar luxation, one (1.3\%) was mixed-breed and 13.3\% were purebred, with high prevalence in Poodles $(n=6 ; 8 \%)$ and Pinschers $(n=2 ; 2.7 \%)$. Regarding the 62 dogs with medial patellar luxation, 20 were unilateral and 42 bilateral. Of the 11 dogs with lateral patellar luxation, five were unilateral and six bilateral. Statistically, females were more affected than males by both medial and lateral patellar luxations. The grade II (25; $33.3 \%)$ of patellar luxation was the most often in medial luxation followed by grades III (20; $26.7 \%), I V$ $(11 ; 14.7 \%)$, and I (16; 7.9\%). The grade II (5; 6.7\%) was the most often in lateral luxation followed by grade I (4; 5.3\%), and simultaneously grades III (1.3\%) and IV (1.3\%). The surgical treatment was performed in 64 dogs and the others were submitted a conservative management. It was possible to conclude that among the dogs with patellar luxation evaluated in this study, the most affected were toy and miniature breeds and female dogs with medial patellar luxation.
\end{abstract}

Keywords: dog, stifle joint, patellar luxation

M.M.D. Souza é bolsista PIBIC/CNPq

\section{AGRADECIMENTOS}

Ao Conselho Nacional de Desenvolvimento Científico e Tecnológico (CNPq).

\section{REFERÊNCIAS BIBLIOGRÁFICAS}

ALAM, M.R.; LEE, J.I.; KANG, H.S. et al. Frequency and distribution of patellar luxation in dogs - 134 cases (2000 to 2005). Vet. Comp. Orthop. Traumatol., v.20, p.59-64, 2007.

ARNOCZKY, S.P.; TARVIN, G.B. Surgical repair of patellar luxations and fractures. In: BOJRAB, M.J.; ELLISON, G.W.; SLOCUM. B. (Eds). Current techniques in small animal surgery. 4.ed. Philadelphia: Lippincott Williams \& Wilkins, 1998. p.1237-1244.

De ANGELIS, M.; HOHN, R.B. Evaluation of surgical correction of canine patellar luxation in 142 cases. J. Am. Vet. Med. Assoc., v.156, p.587-599, 1970.

DENNY, H.R.; BUTTERWORTH, S.J. The stifle. In:__. A guide to canine and feline orthopaedic surgery. 4.ed. Oxford: Blackwell Science, 2000. p.512-553.

GONÇALVES, G.; MELO, E.G.; GOMES, M.G. et al. Effects of chondroitin sulfate and sodium hyaluronate on chondrocytes and extracellular matrix of articular cartilage in dogs with degenerative joint disease. Arq. Bras. Med. Vet. Zootec., v.60, p.93-102, 2008.

HAYES, A.G.; BOUDRIEAU, R.J.; HUNGERFORD, L.L. Frequency and distribution of medial and lateral patellar luxation in dogs: 124 cases (1982-1992). J. Am. Vet. Med. Assoc., v.205, p.716-720, 1994.

HORNE, R.D. Canine patellar luxation (a review). Vet. Med. Small Anim. Clin., v.66, p.211-218, 1971.

HULSE, D.A. Pathophysiology and management of medial patellar luxation in the dog. Vet. Med. Small Anim. Clin., v.76, p.43-51, 1981.

JOHNSON, A.L.; HULSE, D.A. Diseases of the joints. In: FOSSUM, T.W. (Ed). Small animal surgery. St. Louis: Mosby, 2002. p.1023-1157.

LaFOND, E.; BREUR, G.J.; AUSTIN, C.C. Breed susceptibility for developmental orthopedic diseases in dogs. J. Am. Anim. Hosp. Assoc., v.38, p.467-477, 2002. L'EPLATTENIER, H.; MONTAVON, P. Patellar luxation in dogs and cats: pathogenesis and diagnosis. Compend. Contin. Educ. Pract. Vet., v.24, p.234-239, 2002.

PRIESTER, W.A. Sex, size, and breed as risk factors in canine patellar luxation. J. Am. Vet. Med. Assoc., v.160, p.740-742, 1972.

READ, R.A. Opciones racionales de tratamiento de la luxación interna de rótula. Waltham Focus, v.9, p.2531, 1999.

ROUSH, J.K. Canine patellar luxation. Vet. Clin. N. Am.: Small Anim. Pract., v.23, p.855-868, 1993.

TOMLINSON, J.; CONSTANTINESCU, G.M. Repair of medial patellar luxation. Vet. Med., v.89, p.48-56, 1994.

WILLAUER, C.C.; VASSEUR, P.B. Clinical results of surgical correction of medial luxation of the patella in dogs. Vet. Surg., v.16, p.31-36, 1987. 